\title{
Lambda z Lower Limit TAU
}

National Cancer Institute

\section{Source}

National Cancer Institute. Lambda z Lower Limit TAU. NCI Thesaurus. Code C147479.

The lower limit on time for values to be included in the calculation of Lambda $z$, calculated within a dosing interval. 